\title{
Recent advances and perspectives of metabolomics-based investigations in Parkinson's disease
}

\author{
Yaping Shao ${ }^{1,2}$ and Weidong Le 1,2* $^{*}$
}

\begin{abstract}
Parkinson's disease (PD) is the second most prevalent neurodegenerative disease of the central nervous system (CNS), which affects mostly older adults. In recent years, the incidence of PD has been dramatically increasing with the aging population expanding. Due to the lack of effective biomarkers, the accurate diagnosis and precise treatment of PD are currently compromised. Notably, metabolites have been considered as the most direct reflection of the physiological and pathological conditions in individuals and represent attractive candidates to provide deep insights into disease phenotypes. By profiling the metabolites in biofluids (cerebrospinal fluid, blood, urine), feces and brain tissues, metabolomics has become a powerful and promising tool to identify novel biomarkers and provide valuable insights into the etiopathogenesis of neurological diseases. In this review, we will summarize the recent advancements of major analytical platforms implemented in metabolomics studies, dedicated to the improvement and extension of metabolome coverage for in-depth biological research. Based on the current metabolomics studies in both clinical populations and experimental PD models, this review will present new findings in metabolomics biomarkers research and abnormal metabolic pathways in PD, and will discuss the correlation between metabolomic changes and clinical conditions of PD. A better understanding of the biological underpinning of PD pathogenesis might offer novel diagnostic, prognostic, and therapeutic approaches to this devastating disease.
\end{abstract}

Keywords: Parkinson's disease, Metabolomics, Biomarker, Metabolic pathway

\section{Background}

Parkinson's disease (PD) is a progressive, multi-focal neurodegenerative disorder, affecting approximately $1 \%$ of people over the age of $60[1,2]$. The diagnosis of PD mainly relies on clinical symptoms, medical history, and response to dopaminergic treatment, which results in a high rate of misdiagnosis in the clinical practice of $\mathrm{PD}$ $[3,4]$. In addition, the clinical manifestations of PD patients usually lag behind the underlying pathological changes in the brain, making the early diagnosis of PD a great challenge [5]. Currently, the most commonly used therapeutic strategy for PD, dopamine replacement therapy, can only improve the clinical motor symptoms and

\footnotetext{
* Correspondence: wdle@sibs.ac.cn

${ }^{1}$ Center for Clinical Research on Neurological Diseases, The First Affiliated Hospital, Dalian Medical University, Dalian, China

${ }^{2}$ Liaoning Provincial Key Laboratory for Research on the Pathogenic Mechanisms of Neurological Diseases, The First Affiliated Hospital, Dalian Medical University, Dalian, China
}

is incapable of slowing or halting disease progression. Markedly, long-term medical treatment can lead to serious, irreversible motor complications, such as L-dopa induced dyskinesia (LID) [6]. Although, a range of biomarkers derived from clinical, neuroimaging, genetic, and biochemical studies have been proposed [7-12], sensitive, specific, and reliable biomarkers for PD remain elusive. Deterioration of dopaminergic neurons within the substantia nigra pars compacta and the accumulation of intracytoplasmic inclusions known as Lewy Bodies are hallmarks of the PD pathobiology [13]. Currently, the proposed hypotheses for the pathogenesis of PD include protein misfolding and aggregation, mitochondrial injury, oxidative stress and inflammation [14, 15]. However, since PD is a multifactorial disease, it is likely that multiple mechanisms may contribute to its pathogenesis. Despite decades of research, the underlying etiopathogenesis of PD is still not fully elucidated. Given the lack of knowledge regarding the mechanisms that regulate

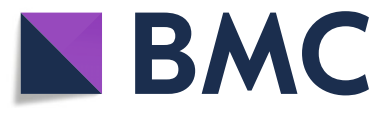

(c) The Author(s). 2019 Open Access This article is distributed under the terms of the Creative Commons Attribution 4.0 International License (http://creativecommons.org/licenses/by/4.0/), which permits unrestricted use, distribution, and reproduction in any medium, provided you give appropriate credit to the original author(s) and the source, provide a link to the Creative Commons license, and indicate if changes were made. The Creative Commons Public Domain Dedication waiver (http://creativecommons.org/publicdomain/zero/1.0/) applies to the data made available in this article, unless otherwise stated. 
the onset and progression of the disease pathology, new approaches dedicated to the discovery of specific biomarkers that offer more accurate diagnosis, and better monitoring of PD progression and prognosis are in urgent need. Furthermore, the identification of reliable targets might lead to the development of novel drugs, which could reverse the neurodegeneration and progression of PD.

Metabolomics is an emerging technique that aims to investigate the global changes of numerous metabolites within a given sample, followed by deep data mining and bioinformatic analysis [16, 17] (Fig. 1). These metabolites are not only endogenous, but are also derived from the metabolism of pharmaceuticals, environmental chemicals, and the co-metabolism between host and gut microbiota [17]. Minor changes of endogenous and exogenous factors can be reflected at the level of metabolites; thus, the study of metabolomics has immense potential to link the genetic, environmental, and physiological elements to specific pathological states [18]. In the past decades, metabolomics has become a powerful tool for investigating metabolic processes, identifying potential biomarkers and unraveling metabolic reprogramming in various diseases [5, 19-21]. Advancements and achievements in both biological sample preparation and instrumental techniques have made the high-throughput analysis of a broad range of metabolites possible, stimulating a great interest regarding its potential application in PD research.

In this review, we summarized major improvements in analytical platforms and recent advancements in metabolomics studies, and discussed the advantage and limitation of each methodology. Then, we reviewed applications of metabolomics in PD research, and discussed the major metabolic findings in the metabolome of cerebrospinal fluid (CSF), blood, urine, feces and brain tissue in clinical populations as well as in experimental PD models. Finally, we outlined several abnormal metabolic pathways in $\mathrm{PD}$, which may improve our knowledge on the molecular mechanisms mediating PD development, which can help develop new therapeutic strategies for this devastating disease.

\section{Major analytical platforms in metabolomics research}

Nuclear magnetic resonance (NMR) spectroscopy and mass spectrometry (MS) are two predominant analytical platforms used in metabolomics [22]. Particularly, chromatograph-MS coupled systems including liquid chromatography-MS (LC-MS) and gas chromatography-MS (GC-MS) are the most popular techniques.

\section{Nuclear magnetic resonance spectroscopy}

NMR is a powerful tool commonly used for the identification of metabolites. It offers various relevant and excellent attributes such as simple sample preparation, short analysis time, robust signal, and absolute quantification of metabolites [23]. However, the relative low sensitivity of NMR makes it incapable of measuring low-abundance metabolites. Due to the absence of a proper separation system, thousands of metabolites signals are overlapped, which make accurate structure identification a complicated and difficult task. Nonetheless, recent technological achievements have minimized these drawbacks and have improved the sensitivity and resolution of NMR techniques; the applications of highly sensitive cryoprobes and
$1 \quad$ Problem formulation

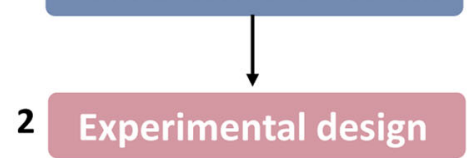

2 Experimental design

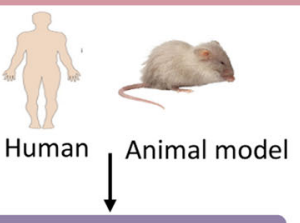

3 Sample collection
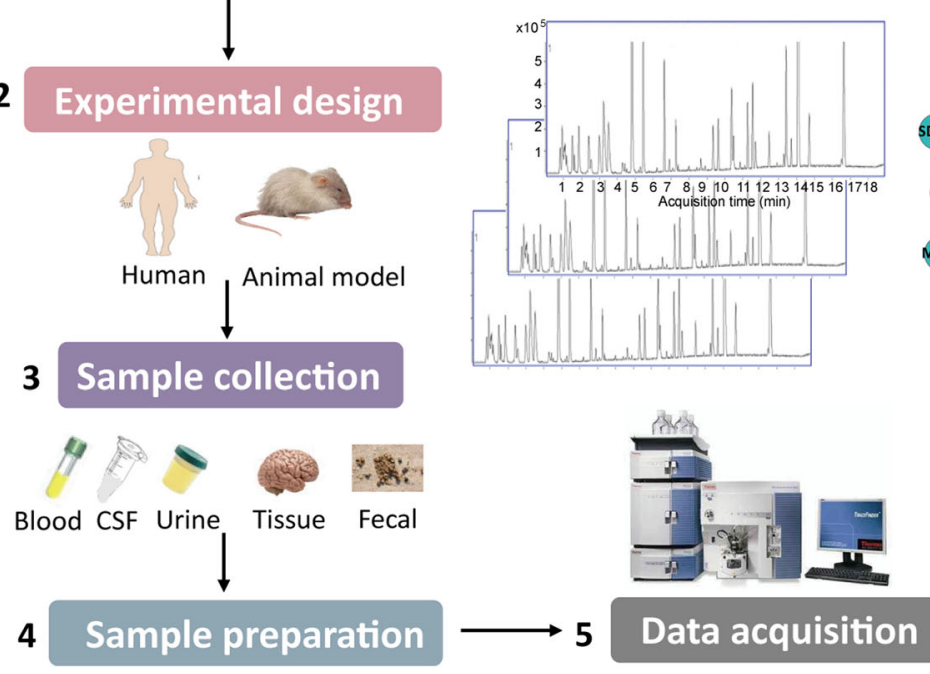

7 Functional interpretation

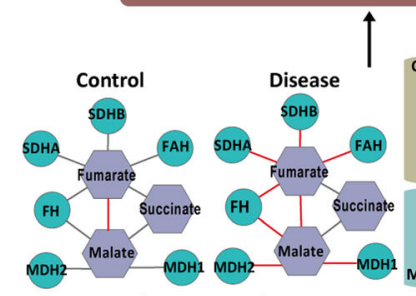

Correlation analysis

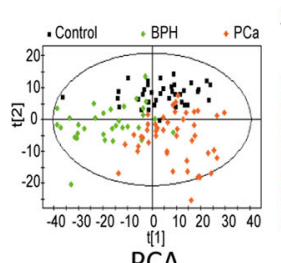

PCA

Statistical analysis

Fig. 1 Analytical workflow of metabolomics studies. The typical metabolomics study including experimental design, sample collection, sample preparation, data acquisition, statistical analysis and functional interpretation stages 
microprobes benefit to detect low-abundance metabolites with the detection limit reduced by $3 \sim 5$ times $[24,25]$. In addition, advanced NMR methods include two-dimensional (2D) NMR techniques, such as HSQC and TOCSY as well as hyphenated LC-MS-NMR, which have made great progress in recent years, improving both spectral resolution and metabolite identification capabilities $[26,27]$.

\section{Gas chromatography-mass spectrometry}

GC-MS has been extensively used in metabolomics, particularly given its high separation power and reliable structure annotation capacity [28]. GC can be coupled to diverse types of mass analyzers, such as single quadrupole $(\mathrm{Q})$, triple quadrupole $(\mathrm{QqQ})$, ion trap (IT), and time of flight (TOF). Recently, the newly developed GC/ Q-Orbitrap MS system has been shown to greatly improve the ability to identify unknown metabolites, due to its higher sensitivity, resolution, and mass accuracy [29]. In addition, chromatography separation techniques have been also improved. By combining two orthogonal columns, 2D GC yielded a multiplicative increase in peak capacity [30].

GC-MS is mainly used to analyze volatile (i.e., naturally volatile and made volatile by derivatization) and thermally stable metabolites. Among a multitude of chemical derivatization methods, a two-step process that includes oximation followed by trimethylsilylation, provides a broad coverage of metabolites and is currently the most commonly used approach [31]. The metabolites detected by GC-MS are mainly associated with tricarboxylic acid (TCA) cycle, glycolysis, urea cycle, amino acid metabolism, and fatty acid metabolism, among others. Recently, a group of fast and sensitive GC-MS-based methods have been developed for the quantification of short-chain and medium-chain fatty acids, and proved to be effective tools for exploring the effects of host-gut microbiota [32, 33]. GC-MS has been also used to explore the dysregulation of neurotransmitter, hormones, and purine metabolism in different neurological diseases [34, 35].

\section{Liquid chromatography-mass spectrometry}

LC-MS is a widely used analytical platform in metabolomics research. Reverse-phase liquid chromatography (RPLC) and hydrophilic interaction liquid chromatography (HILIC) are two major chromatographic separation techniques [36], which provide complementary metabolic information [37]. Recently, 2D and multidimensional LC have emerged as powerful analytical techniques that provide higher peak capacity and better resolution by combining two or more columns with orthogonal characteristics [36]. The newly established 2D-LC-MS method enables the simultaneous analysis of metabolome and lipidome in one single run and is viewed as an efficient tool for large-scale metabolomics studies with a limited amount of samples [38].

Untargeted and targeted analyses are two traditional strategies for metabolomics studies [39]. Untargeted metabolomics has the best metabolites coverage, however it holds poor reproducibility and limited sensitivity for low-abundance metabolites [40]. Targeted metabolomics has been regarded as the gold standard for metabolite quantification due to its high sensitivity, broad dynamic range, and reliable quantification accuracy, although it covers limited pre-known metabolic information [41]. Dynamic multiple reaction monitoring (MRM)-based pseudo-targeted metabolomics quantification and parallel reaction monitoring (PRM)-based larger-scale targeted metabolomics quantification, are two newly emerged strategies, both of which can measure a large number of metabolites with reliable quantitative arrays and are now proved to be powerful tools for metabolomics studies [42].

Although all of these techniques enable simultaneous identification and quantitation of multitudinous metabolites coexisting in one single sample, none of them are able to cover the entire metabolome yet. Nevertheless, the combination of multiple analytical platforms can contribute to an improved metabolic coverage.

\section{Metabolomics studies in patients with PD}

Initially, traditional targeted approaches were mainly implemented in the evaluation of a few selected metabolites of interest, including catecholamines, amino acids, purines and urate [43-45]. Until the last decade, untargeted metabolomics has been applied to PD research, counting on its enormous potential for the identification of novel biomarkers. Most of these studies are based on CSF and blood analysis, although some studies have examined other biological samples such as urine, feces or brain tissue. In the following section, we will review primary metabolomics-based findings in the metabolome of different sample matrices obtained from PD patients.

\section{Cerebrospinal fluid metabolome}

CSF composition abnormalities are directly related to pathological changes in the brain, making CSF one of the preferred samples for neuropathological studies. Given the marked depletion of the nigrostriatal dopaminergic neurotransmission in PD patients, measurements of dopamine levels and its metabolites may provide a path to the discovery of a reliable trait biomarker [43]. Using the LC- electrochemistry array (LCECA) based targeted approach, significant reduction of catecholamines including homovanillic acid [46] (HVA), dihydroxyphenylacetic acid (DOPAC), L-dopa, and dihydroxyphenylglycol has been reported in $\mathrm{PD}$ [43]. Among others, DOPAC levels showed high accuracy in distinguishing PD (including early onset) from 
controls. However, low levels of DOPAC are not specific for PD, and a marked reduction of catechols has been also observed in patients with other synucleinopathies like pure autonomic failure and multiple system atrophy (MSA) [43].

Other metabolites of interest in the CSF of PD are purines. An exploratory study investigating the levels of xanthine and HVA in PD versus controls using the LCECA platform, found that the ratio of xanthine to HVA in CSF permitted an excellent distinction of PD from controls [47]. In addition, statistically significant high-levels of 8-hydroxy-2-deoxyguanosine (8-OHdG) and 8-hydroxyguanosine (8-OHG) were observed in PD, when compared to controls in two independent studies [48, 49], indicating that oxidative stress markers could be potentially useful in the diagnosis of PD.

The wide metabolites coverage and high-throughput analysis of untargeted metabolomics make it an effective tool for the discovery of novel PD biomarkers. Using GC-TOF/MS-based metabolomics, significant reductions in tryptophan, creatinine, and 3-hydroxyisovalerate levels were reported in PD compared to controls [50]. Another study based on NMR metabolomics further identified a panel of metabolites (alanine, creatinine, dimethylamine, glucose, lactate, mannose, phenylalanine, 3-hydroxyisobutyric acid and 3-hydroxyisovaleric acid) that exhibited a good capacity to discriminate PD from controls [51]. Recently, using untargeted MS-driven approach, specific metabolic signatures of PD in early stages of the disease were uncovered $[5,52]$. These PD-specific metabolites have been shown to be involved in antioxidative stress responses, and metabolic pathways of sphingolipid, glycerophospholipid and amino acid, which may aid in the accurate diagnosis of early-stage PD [5, 52]. It was noteworthy that Stoessel et al. demonstrated a relatively high overlapping of metabolome in CSF and blood, implying a joint analysis of multiple biofluids collected from the same subject will be more valuable in reflecting the overall metabolism [52].

\section{Blood metabolome}

Compared with CSF metabolomics research, a larger number of untargeted metabolomics-based studies have been reported using plasma/serum samples, possibly due to its minimally invasive nature and relatively easy availability of blood samples. We summarized the major findings of blood metabolome studies of PD published over the past decade (Table 1). In general, case-control studies accounted for the majority, except for several studies including subgroups of PD such as LRRK2 mutation [16] and patients with or without LID [6]. The differential metabolites between PD and matched controls can be classified as amino acids, fatty acids, acylcarnitines, lipids, purines, organic acids and sugars, which are parts of branched chain amino acids (BCAAs) metabolism, tryptophan metabolism, lipid metabolism, energy metabolism, purine metabolism, and oxidative stress/ redox homeostasis pathways. Recently, a group of studies consistently demonstrated the dysregulation of kynurenine pathway in PD [19, 53, 54]. The alterations of kynurenine metabolites in PD not only provide potential biomarker candidates and novel avenues for investigating PD pathogenesis, but also offer a new therapeutic strategy for PD with the supplement of kynurenic acid or the reduction of quinolinic acid using kynurenine 3-monooxygenase inhibitors [19].

Plenty of biological, epidemiological and clinical studies have convergently suggested urate as a promising biomarker of the risk, diagnosis and prognosis of PD. Significantly reduced level of urate in both CSF and blood of PD was reported compared to controls [45, 55], and a high level of urate may indicate a lower risk, slower progress of the disease [55-57]. As an important endogenous antioxidant [55], increased level of urate may contribute to fight against oxidative stress in the pathogenesis of PD [58]. Detailed elaborations of the correlation between urate and PD have been reviewed elsewhere [55, 59].

As previously mentioned, PD is a multifactorial disease with compelling epidemiological data that suggest a probable link between traumatic brain injury (TBI) and PD; however, such association is still controversial due to the lack of mechanistic basis [60]. Based on untargeted and targeted LC-MS approaches, a statistically significant alteration of glutamate level was identified in blood samples from both TBI and PD, implying a possible "excitotoxic" link between TBI and PD [61]. Additionally, the overlap of clinical symptoms between PD and other neurodegenerative diseases, such as primary progressive multiple sclerosis (PPMS), progressive supranuclear palsy (PAP) and MSA often lead to high rates of misdiagnosis for PD [3]. Recently, two studies using NMR and LC-MS based metabolomics profiled the blood metabolome of patients with PD, PPMS, PAP, and MSA versus controls, showing that BCAAs were significantly increased in PD, PAP, and MSA compared to controls [3], and a set of 20 metabolites involved in glycerophospholipid and linoleic acid pathways were specifically altered in PPMS which were distinguishable from PD [48].

Metabolomics can also reveal biomolecular and pathway changes implicated in disease onset and progression. To this end, it has been reported that the level of N8-acetyl spermidine may be a predictive marker for a fast motor progression disease phenotype, which may provide a novel strategy for delaying or slowing down the progression of PD [49]. Based on metabolomics approaches, plasma metabolic profiles of serine, purine, 
Table 1 Overview of metabolomic studies in the blood metabolome of PD clinical populations

\begin{tabular}{|c|c|c|c|c|c|}
\hline Analytical platform & Subjects & Differential metabolites/metabolic pathways & Statistics & Validation & Reference \\
\hline HILIC-TOF/MS & $\begin{array}{l}\text { Early PD }(n=80) \\
\text { Controls }(n=76)\end{array}$ & $\begin{array}{l}\text { Ethanolamine, N-Lauroylglycine, } \\
\text { Alpha-N-Phenylacetyl-L-glutamine, } \\
\text { Sarcosine, Glu-lle, 1,3-Dimethyluracil, } \\
\text { Arg-Ala, PCs, SMs, Lyso-PAF C-16, etc. }\end{array}$ & $\begin{array}{l}\text { ROC } \\
(A \cup C=0.80)\end{array}$ & No & Stoessel D et al. [52] \\
\hline $\begin{array}{l}\text { CE-TOF/MS } \\
\text { LC-TOOF/MS }\end{array}$ & $\begin{array}{l}\text { PD }(n=109) \\
\text { Controls }(n=32)\end{array}$ & Long chain acylcarnitines & $\begin{array}{l}\text { ROC } \\
(A \cup C=0.895)\end{array}$ & Yes & Saiki S et al. [102] \\
\hline LC-MS & $\begin{array}{l}\text { PD }(\operatorname{LID}, n=10 \\
\text { non-LID, } n=8 \\
\text { unmedicated, } n=8) \\
\text { Controls }(n=14)\end{array}$ & $\begin{array}{l}\text { 3-hydroxykynurenine/kynurenic acid } \\
\text { Ratio }\end{array}$ & t-test, $p<0.05$ & No & Havelund JF et al. [6] \\
\hline $\begin{array}{l}\text { Nontargeted MS-based } \\
\text { metabolomics }\end{array}$ & $\begin{array}{l}\text { Early PD }(n=41) \\
\text { Controls }(n=40)\end{array}$ & $\begin{array}{l}\text { Hexanoylglutamine, Decanoylcarnitine, } \\
\text { Myristoleoylcarnitine, Octanoylcarnitine, } \\
\text { Oleoylcarnitine, Palmitoleoylcarnitine, } \\
\text { Suberoylcarnitine, Octadecanedioate, } \\
\text { 3-hydroxysebacate }\end{array}$ & $\begin{array}{l}\text { ROC } \\
(A \cup C=0.857)\end{array}$ & No & Burté F et al. [111] \\
\hline $\begin{array}{l}\text { UPLC-MS/MS } \\
\text { GC-MS }\end{array}$ & $\begin{array}{l}\text { PD }(n=35) \\
\text { Controls }(n=15)\end{array}$ & $\begin{array}{l}\text { Lower levels of tryptophan, caffeine, bilirubin } \\
\text { and ergothioneine; higher levels of levodopa } \\
\text { metabolites and biliverdin }\end{array}$ & $\begin{array}{l}\text { random forest } \\
\text { classification }\end{array}$ & No & Hatano T et al. [112] \\
\hline NMR & $\begin{array}{l}\text { PD }(n=43) \\
\text { Controls }(n=37)\end{array}$ & $\begin{array}{l}\text { Myoinositol, sorbitol, citrate, acetate, succinate } \\
\text { and pyruvate }\end{array}$ & PLS-DA & No & Ahmed SS et al. [113] \\
\hline LCECA & $\begin{array}{l}\text { LRRK2 PD }(n=12) \\
\text { idiopathic PD }(n=41) \\
\text { Controls }(n=46)\end{array}$ & $\begin{array}{l}\text { Purine metabolism (uric acid, hypoxanthine, } \\
\text { xanthine, etc.) }\end{array}$ & PLS-DA & No & $\begin{array}{l}\text { Johansen KK et al. } \\
\text { [16] }\end{array}$ \\
\hline LCECA & $\begin{array}{l}\text { PD }(n=66) \\
\text { Controls }(n=25)\end{array}$ & 8-OHdG, glutathione, uric acid & PLS-DA & No & $\begin{array}{l}\text { Bogdanov } M \text { et al. } \\
{[17]}\end{array}$ \\
\hline GC-TOFMS & $\begin{array}{l}\text { PD }(n=20) \\
\text { Controls }(n=20)\end{array}$ & $\begin{array}{l}\text { Amino acids (pyroglutamate and } \\
\text { 2-oxoisocaproate), C16-C18 saturated and } \\
\text { unsaturated fatty acids }\end{array}$ & OPLS-DA & No & Trupp M et al. [50] \\
\hline $\begin{array}{l}\text { LC-MS } \\
\text { GC-MS }\end{array}$ & $\begin{array}{l}\mathrm{PD}(n=82) \\
\mathrm{RLS}(n=95) \\
\text { Controls }(n=1272)\end{array}$ & $\begin{array}{l}\text { Long-chain (polyunsaturated) fatty acids, } \\
\text { inositol metabolites }\end{array}$ & & No & Kassubek J et al. [101] \\
\hline UPLC-TOF/MS & $\begin{array}{l}\text { PD (cohort } 1, n=82, \\
\text { cohort } 2, n=118 \text { ) } \\
\text { Controls (cohort } 1, n=82, \\
\text { cohort } 2, n=37 \text { ) } \\
\text { Huntington's disease } \\
\text { (cohort2, } n=22 \text { ) }\end{array}$ & $\begin{array}{l}\text { Kynurenic acid, quinolinic acid, ratio of } \\
\text { kynurenic acid/kynurenine, ratio of } \\
\text { quinolinic acid/ kynurenic acid }\end{array}$ & OPLS-DA & Yes & Chang KH et al. [19] \\
\hline LC-QE/MS & $\begin{array}{l}\text { Slow. PD }(n=41) \\
\text { Rapid. PD }(n=39) \\
\text { Controls }(n=20)\end{array}$ & N8-acetyl spermidine & OPLS-DA & No & Roede JR et al. [49] \\
\hline
\end{tabular}

fatty acid, polyamines, and metabolites associated with tryptophan metabolism have presented a high correlation with the progression of PD [6, 19, 53]. Additionally, it has been shown that kynurenine metabolism is also associated with the development of LID, and increased plasma ratio of 3-hydroxykynurenine /kynurenic acid may predict the possibility of LID $[6,19]$.

\section{Urine metabolome}

Given the easy availability and noninvasive sampling of urine samples, they are ideal sources of biomarkers for clinical analysis. Incipiently, research studies were focused on evaluating oxidative stress markers by targeted analysis strategies [62-64]. Under the attack of reactive oxidative species, the bases in DNA can be hydroxylated and oxidized; 8-OHdG and 8-OHG are two of the most prominent products of DNA impairment [65]. The resulting 8 -OHdG can be excreted into urine without further metabolism, which is regarded as an indicator of oxidative DNA damage [63]. Previous studies demonstrated elevation of 8-OHdG level in the substantia nigra of the brain $[66,67]$ as well as in the serum and CSF of PD [68]. Based on targeted analysis, it was demonstrated that level of $8-\mathrm{OHdG}$ in the urine alone or the ratio of 8-OHdG/2' -deoxyguanosine can significantly distinguish PD from the controls [63]. In addition, level of urinary 8-OHdG showed a progressive increase with PD advances, suggesting that it may be an useful biomarker to track disease progression [62]. Moreover, utilizing non-targeted metabolomic profiling method, biopyrrin was identified as a new marker for sporadic PD [69]. Biopyrrin, the oxidative product of bilirubin, has been 
regarded as the indicator of increased oxidative stress, showed high predictable ability for different stages of PD (AUC $=0.95 \sim 0.98)$ [69].

The urine, which contains abundant metabolites, has seldom been investigated by untargeted metabolomics in PD research. Using LC-MS and a random forest model, a recent study profiled urinary metabolites in sporadic $\mathrm{PD}$ versus controls and identified a panel of metabolites that yielded with $>90 \%$ accuracy in distinguishing PD from controls [70]. Based on GC-MS and LC-MS technologies, another study of the same group identified 18 metabolites that showed progressive increases with the development of PD [71]. Both of these studies indicate that the dysregulation of steroidogenesis, glycine derivation, tryptophan and phenylalanine metabolic pathways are related to the development and progression of $\mathrm{PD}$ [71]. Recently, an assay combining UPLC-MS/MS with in situ selective derivatization was developed to detect a wide range of neurochemicals in urine samples, presenting a promising analytical platform to screen potential biomarkers that can aid in the diagnostic accuracy and tracking of PD prognosis [72].

\section{Fecal metabolome}

Recent investigations have highlighted the crucial role of the gut microbiota in the development of neurodegenerative diseases including PD [73-75]. Fecal metabolome, can provide information regarding the metabolic interactions between host, diet, and gut microbes, presenting a promising avenue to "fingerprint" the functional status of the intestinal microbiota and explore links between microbiome and host phenotypes [76]. Fecal metabolomics has been widely used in both biomarker identification and functional annotation for various diseases, such as irritable bowel syndrome, nonalcoholic fatty liver disease, obesity, and autism [77-79]; however, this approach has been rarely used for the investigation of neurodegenerative diseases. Recently, a reduction of fecal short chain fatty acids (SCFAs) was identified in $\mathrm{PD}$, when compared to controls by GC-based quantitative analysis [80]. Since SCFAs can regulate the function of the enteric nervous system and promote gastrointestinal motility, a reduction of SCFAs might contribute to the development of gastrointestinal motility disorders in PD [80, 81].

\section{Tissue metabolome}

Human brain metabolomics studies are mainly based on NMR spectroscopy techniques, which enable non-destructive detection of the chemical composition of a specific area in a living body. In vivo PD brain metabolomics based on NMR spectroscopy has been reviewed elsewhere [82]. In summary, these studies are mainly focused on the mitochondrial dysfunctions observed in PD patients by tracing the levels of creatine, phosphocreatine, ATP, high-energy phosphates, phospholipids, and lactate [82-85], and indicate impaired mitochondrial oxidative phosphorylation events in the brain of PD patients, even in the absence of a clinical phenotype. Moreover, the mitochondrial machinery in patients carrying a PINK1 mutation, was more susceptible to these events than idiopathic PD [86]. Notably, the combined evaluation of $\mathrm{N}$-acetylaspartate/creatine levels from both the pontine base and putamen in brain tissues may offer effective strategies to distinguish MSA with predominant Parkinsonism from PD, as reported by a number of studies [82, 87, 88]. By implementing a LC-MS-based lipidomics technique, a recent study identified abnormal levels of diacylglycerols in the frontal cortex of PD patients who presented no neocortical pathology [89]. These data suggest that the elevation of plasma levels of diacylglycerols in PD may be a promising marker for neurodegenerative processes that ought to be further investigated.

\section{Metabolomics studies in PD models}

Although various types of animal models have been established for PD research, only a few of them have been used for metabolomics studies. We summarized these studies in Table 2, the genetic models used include $\alpha$-synuclein $(\alpha-$ Syn) knockout, $\alpha$-Syn transgenic, $\alpha$-Syn overexpressed [90-92] and Park2 knockout animal models [93], while toxicological models are mainly induced by paraquat, rotenone, 1-methyl-4-phenyl-1, 2,3,6-tetrahydropyridine (MPTP), methyl-4-phenylpy ridinium, and 6-hydroxydopamine (6-OHDA) [94-96]. In these animal studies, the metabolic profiles identified originated primarily from brain tissues (whole brain or specific areas), which better reflect the patho-physiological changes.

Recently, a mouse model of prodromal PD was established via unilateral injection of preformed $\alpha$-Syn fibrils in the olfactory bulb [97]. Contrary to earlier reports, both brain tissue and serum were collected and subjected to metabolomics analysis for the development of early diagnostic markers of PD. The pathway enrichment of the brain data suggested a dysregulation of taurine and hypotaurine metabolism, bile acid biosynthesis, glycine, serine and threonine metabolism and the TCA cycle were in correlation with the onset and progression of $\alpha$-Syn pathology, while the results from the serum highlighted only phospholipid metabolism alterations in $\alpha$-Syn PFF-injected mice that may provide evidence for the possible interaction between lipid metabolism and $\alpha$-Syn aggregation [97].

Consistently, studies from three different groups, although, based on different animal models, showed a relatively limited impact of the genotype on metabolites levels, when compared to aging or toxic exposure [90, 91, 93]. It 
Table $\mathbf{2}$ Overview of metabolomic studies in experimental models of PD

\begin{tabular}{|c|c|c|c|}
\hline Analytical platform & Models & Differential metabolites/metabolic pathways & Reference \\
\hline GC-MS & PQ-exposed Drosophila & Amino acids, fatty acids, carbohydrates, etc. & Shukla AK et al. [21] \\
\hline NMR, DI-ESI-MS & PQ-exposed dopaminergic cell & $\begin{array}{l}\text { Pentose phosphate pathway (PPP), } \\
\text { glycolysis, TCA cycle }\end{array}$ & Lei S et al. [98] \\
\hline NMR & MPTP-induced PD goldfish & $\begin{array}{l}\text { BCAAs, alanine, myo-inositol, fatty acids, taurine, } \\
\text { creatinine, N-acetylaspartate, (phospho)creatine, } \\
\text { phosphatidylcholines, cholesterols, }\end{array}$ & Lu Z et al. [114] \\
\hline LC-MS & Rotenone-treated rats & Oxidizable PUFA-containing cardiolipin & Tyurina YY et al. [99] \\
\hline HPLC-ESI-MS/MS & 6-OHDA-induced rats & $\begin{array}{l}\text { Phosphatidylcholine and lysophosphotidylcholine } \\
\text { lipid }\end{array}$ & Farmer K et al. [100] \\
\hline MS-based lipidomics & a-Syn KO, a-Syn TG mice & Age-related phospholipids & Rappley I et al. [90] \\
\hline NMR, LC-MS & Mouse model of prodromal PD & $\begin{array}{l}\text { Taurine and hypotaurine metabolism, bile acid } \\
\text { biosynthesis, glycine, serine, and threonine } \\
\text { metabolism, and citric acid cycle }\end{array}$ & Graham SF et al. [97] \\
\hline NMR, DIESI-MS & PQ -induced dopaminergic N27 cells & Glucose metabolism & Anandhan A et al. [92] \\
\hline NMR & 6-OHDA-induced rats & $\begin{array}{l}\text { GABA, Glu, Gln, lactate, N-acetylaspartate, } \\
\text { creatine, taurine, and myo-inositol. }\end{array}$ & Zheng $\mathrm{H}$ et al. [115] \\
\hline UPLC-QTOF-MS & MPTP-induced PD mice & $\begin{array}{l}\text { Tyrosine metabolism, mitochondrial beta-oxidation } \\
\text { of long chain saturated fatty acids, fatty acid } \\
\text { metabolism, methionine metabolism, and } \\
\text { sphingolipid metabolism }\end{array}$ & Li XZ et al. [116] \\
\hline UPLC-MS & a-Syn A53T TG mice & $\begin{array}{l}\text { Alanine metabolism, redox and acetyl-CoA } \\
\text { biosynthesis pathways }\end{array}$ & Chen X et la. [91] \\
\hline LC-MS & Park2 kO mice, CCCP-treated mice & Energy metabolism & Poliquin PO et al. [93] \\
\hline
\end{tabular}

was found that lipidomic profiles were age-dependent in the wild type-mice, and the $\alpha$-Syn genotype-dependent phospholipid differences indicated a strong interaction of age and $\alpha$-Syn gene dosage [90]. Based on metabolomics and mathematical model, Poliquin et al. investigated and compared the energy deregulation in the cerebral tissue of genetic (Park2 knockout) and CCCP-induced models of $\mathrm{PD}$, and the findings suggested that genetic perturbations are not sufficient to lead to significant metabolic changes compared to toxin exposure [93].

Toxicological models can, to some extent, simulate the roles of oxidative stress, mitochondrial dysfunction, and dopamine metabolism associated with the pathogenesis of PD, which may contribute to $\alpha$-Syn misfolding and aggregation [98]. A generally profound reduction of lipid species was found in brain tissues of PD models induced by rotenone and 6-OHDA, except for a few lipids that showed elevated levels, such as mono-oxygenated cardiolipins (CLs) [99] and several lysophosphatidylcholines [100], all pointing to an increased oxidative damage, insufficient energy and mitochondrial dysfunction in PD. In contrast, the most striking metabolic alterations induced by paraquat treatment were the selective up-regulation of the pentose phosphate pathway (PPP) and the down-regulation of the glycolysis and the TCA cycle $[92,98]$. Powers et al. indicated that the alterations in energy metabolism were not bystanders to energy failure, but also played important roles in dopaminergic cell death via gene ( $\alpha$-Syn)-environment (paraquat) interactions [92].

Metabolic alteration differences among the various genetic/toxic-induced models highlight the multifactorial nature of PD. Future longitudinal metabolic profiling studies based on representative animal models will be able to contribute to a better understanding of the onset and development of the disease.

\section{Dysregulation of metabolic pathways in PD}

PD exhibits high heterogeneity, having multiple pathways and molecular mechanisms mediating its molecular pathogenesis. Based on metabolomic findings in clinical and experimental models, the metabolic pathways that are majorly perturbed in PD are related to the metabolism of lipids, energy (TCA cycle, glycolysis, PPP, BCAA, acylcarnitines), fatty acids, bile acids, polyamine, and amino acids (Fig. 2) [49, 50, 90, 98, 99, 101, 102].

Markedly, a significant reduction of catecholamine metabolite level has been shown for both PD patients and PD animal models, due to the marked depletion of nigrostriatal dopaminergic neurons in PD pathology. The treatment with the different dopaminergic drugs available could selectively increase the levels of these metabolites [103]. In addition, L-DOPA treatment has been also shown to have a profound impact on aromatic amino acid metabolic pathways. Notably, kynurenine metabolism, a pathway of tryptophan metabolism, may 


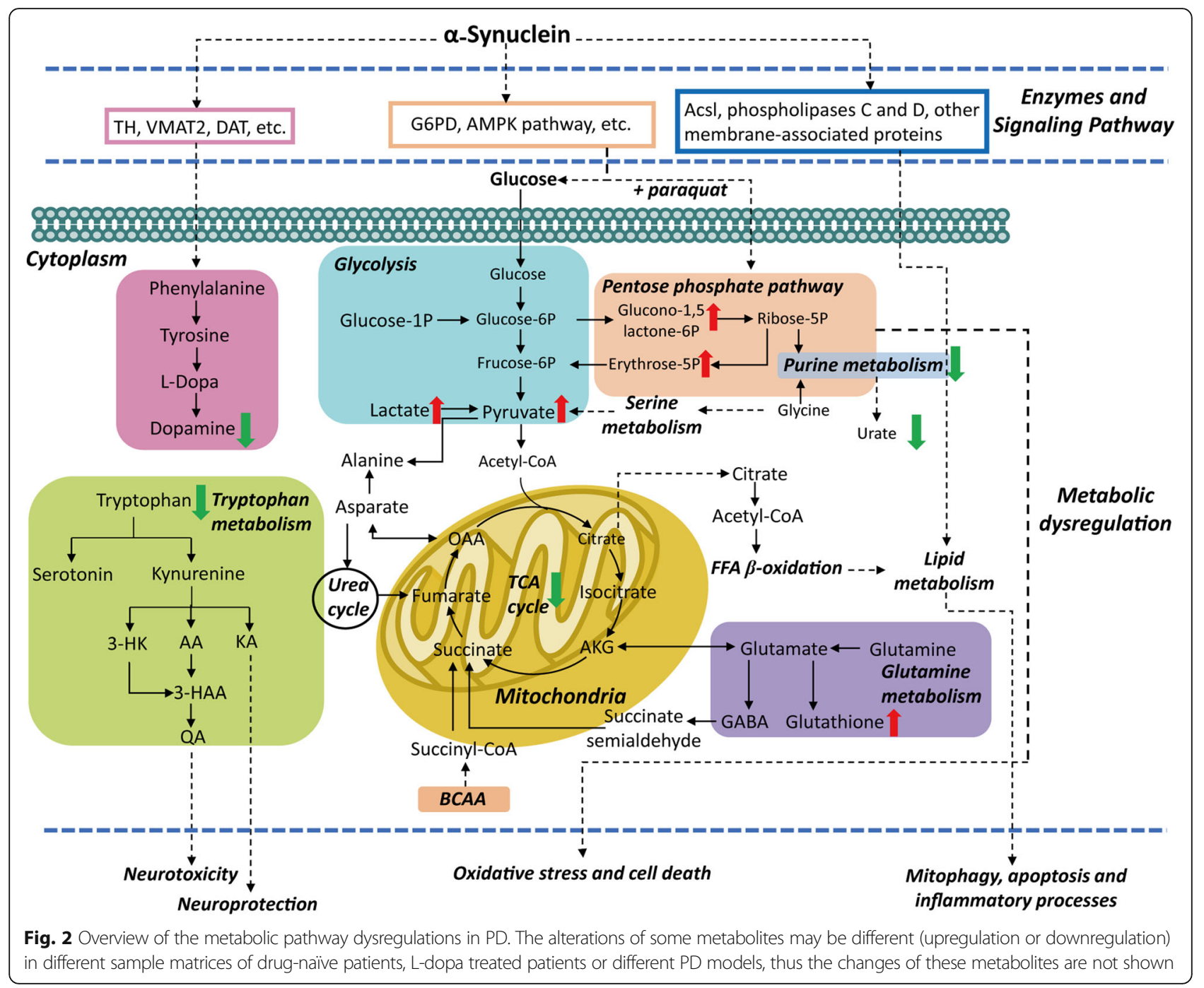

have a strong link with PD progression and the risk of LID development $[6,53]$.

Moreover, accumulating evidence have corroborated that $\alpha$-Syn plays an important role in the pathogenesis of $\mathrm{PD}$ via lipid binding, regulating the composition of membrane, modulating fatty acid metabolism and influencing the release of the neurotransmitter by interacting with specific lipids $[90,104]$. The general reduction of lipids levels, such as polyunsaturated fatty acids (PUFAs) and phospholipids in PD models, presumably due to an excess of oxidative stress, given that membrane phospholipids are major targets for free radicals. The alterations of PUFA-CLs and oxidized CLs not only point to mitochondrial dysfunction, but also indicate possible mitophagy and apoptosis processes in the development of PD [99].

In normal brain function, threonine and glycine can be converted into creatine, which in turn provides phosphate groups for ADP to produce ATP [97]. TCA cycle is an important pathway in the production of ATP through the oxidative phosphorylation of acetyl-CoA in the mitochondrial. With the beginning of $\alpha$-Syn aggregation during the onset of the neurodegenerative processes in PD, the metabolism of glycine, serine, and threonine, as well as the TCA cycle, appear to be downregulated [97], which indicate an energy insufficient and mitochondrial dysfunction in PD. In paraquat-induced models, central carbon metabolism has been shown to contribute to dopaminergic cell death by regulating the effect of $\alpha$-Syn on paraquat toxicity, and inhibiting the metabolism and transport of glucose and PPP can reduce paraquat-induced oxidative stress and cell death [92, 98, 105].

Furthermore, alteration of bile acids has been found in both PD patients and PD animal models [97, 102]. Bile acids are produced in the liver from cholesterol and then metabolized by gut microbiota-derived enzymes into secondary bile acids such as ursodeoxycholic acid or 
tauroursodeoxycholic acid [106]. In addition, it has been demonstrated that tauroursodeoxycholic acid can rescue mitochondrial function and prevent MPTP-induced dopaminergic cell death in different animal models of PD [107].

Currently, the drugs designed to treat or prevent PD are focused on the prevention or elimination of $\alpha$-Syn aggregation; however, no successful cases have been reported yet. In contrast, an alternative and more effective strategy may be the development of specific inhibitors/ activators designed to directly target metabolic processes $[108,109]$. Importantly, metabolomics studies can provide comprehensive biochemical underpinnings to unravel the molecular mechanisms of PD pathogenesis, offering biomarkers that reflect pathological processes and may substantially improve drug development strategies against $\mathrm{PD}$.

\section{Conclusions}

\section{Merits and caveats of metabolomics for PD research}

Metabolic changes are the direct results of alterations in protein and enzyme activities. Therefore, metabolomics may offer valuable information on PD-related physiological process, molecular interactions and metabolic pathways. By providing an overall "fingerprint" of metabolite alterations in multiple biofluids and tissues, metabolomics has provided a myriad of potential biomarkers and therapeutic targets. Nevertheless, metabolomics is still in its infancy, particularly when it comes to PD research. The identification of the unknown metabolites is one of the major challenges. Although great progress has been made during the last decade, the public and commercial databases of metabolites are still limited and incomplete, the current metabolic findings may be only the "tip of the iceberg" of the whole picture of PD etiology. Another important issue is the heterogeneous nature of the individuals. Differences in genotype, medical history, disease progression, lifestyle and diet, etc. of the subjects are likely to affect their metabolome, which may obscure the direct influence from the disease. Besides, the reported works usually used different analytical techniques and different sample preparation methods based on different designs, thus it is not surprised to obtain controversial conclusions.

\section{Future perspectives}

Confirmatory studies based on optimized experimental protocols are urgently needed. The potential biomarkers and metabolic pathways revealed in the present studies require to be validated by independent large-scale populations. As highlighted above, further stratification of PD may allow the identification of specific targets among the different subtypes of PD. Also, a joint analysis of multiple biofluids and tissues using complementary analytical platforms should be employed in parallel to reveal the "bigger picture" for an in-depth biological investigation. It is noteworthy that other related diseases that have similar clinical symptoms with PD should be included in future studies. Identifying metabolites that are specifically changed in PD compared with controls and other related diseases will be of great significance for clinical differential diagnosis. In addition, accumulating evidences suggest that microbiome dysbiosis and changes in microbial metabolite levels are strongly associated with the pathogenesis of PD [74, 75]. Several metabolites involved in the regulation of brain function have been found in the gut, the concentrations of which can be regulated by gut microbiota, further influencing the function of neurons [110]. Given that metabolomics has been shown to be a powerful tool to fingerprint metabolic profiles in multiple matrices, the combination of metabolomics with other techniques, such as metagenomics, proteomics, and transcriptomics may lead to a better understanding of host-microbe interactions and yield potential novel biomarkers for PD diagnosis and therapeutic targets for effective treatment options.

\section{Abbreviations \\ 6-OHDA: 6-hydroxydopamine; 8-OHdG: 8-hydroxy-2-deoxyguanosine; 8- OHG: 8-hydroxyguanosine; AUC: Area under the curve; BCAA: Branched chain amino acid; CE: Capillary electrophoresis; CL: Cardiolipin; CSF: Cerebrospinal fluid; DOPAC: Dihydroxyphenylacetic acid; ECA: Electrochemical detection; GC: Gas chromatography; HVA: Homovanillic acid; IT: Ion trap; LC: Liquid chromatography; LID: L-dopa induced dyskinesia; MPTP: 1-methyl-4-phenyl- 1,2,3,6-tetrahydropyridine; MS: Mass spectrometry; MSA: Multiple system atrophy; NMR: Nuclear magnetic resonance; PAP: Progressive supranuclear palsy; PD: Parkinson's disease; PLS-DA: partial least squares-discriminate ana- lysis; PPMS: Primary progressive multiple sclerosis; Q: Quadrupole; QqQ: Triple quadrupole; RPLC: Reverse-phase liquid chromatographyHILICHydrophilic interaction liquid chromatography; SCFAs: Short chain fatty acids; TBI: Traumatic brain injury; TCA: Tricarboxylic acid; TOF: Time of flight; a- Syn: a-synuclein}

Funding

This study was supported by National Natural Science Foundation of China (81430021, 81771521, 31700853) and Dalian Medical Science Research Project (1812008).

\section{Availability of data and materials Not applicable.}

\section{Authors' contributions}

WL and YS conceived and designed the manuscript. YS did the literature search, writing and editing of the article. WL revised the manuscript, providing expertise in molecular neurodegeneration in PD. Both authors read and approved the final manuscript.

Ethics approval and consent to participate

Not applicable.

Consent for publication

Not applicable.

Competing interests

The authors declare that they have no competing interests. 


\section{Publisher's Note}

Springer Nature remains neutral with regard to jurisdictional claims in published maps and institutional affiliations.

Received: 8 November 2018 Accepted: 6 December 2018

Published online: 11 January 2019

\section{References}

1. de Lau LM, Breteler MM. Epidemiology of Parkinson's disease. Lancet Neurol. 2006;5:525-35.

2. Havelund J, Heegaard N, Færgeman N, Gramsbergen J. Biomarker research in Parkinson's disease using metabolite rofiling. Metabolites. 2017;7:42.

3. Nagesh Babu G, Gupta M, Paliwal VK, Singh S, Chatterii T, Roy R. Serum metabolomics study in a group of Parkinson's disease patients from northern India. Clin Chim Acta. 2018;480:214-9.

4. Rizzo G, Copetti M, Arcuti S, Martino D, Fontana A, Logroscino G. Accuracy of clinical diagnosis of Parkinson disease. Neurology. 2016;86:566.

5. Trezzi JP, Galozzi S, Jaeger C, Barkovits K, Brockmann K, Maetzler W Berg D, Marcus K, Betsou F, Hiller K, Mollenhauer B. Distinct metabolomic signature in cerebrospinal fluid in early parkinson's disease. Mov Disord. 2017;32:1401-8

6. Havelund JF, Andersen AD, Binzer M, Blaabjerg M, Heegaard NHH, Stenager E, Faergeman NJ, Gramsbergen JB. Changes in kynurenine pathway metabolism in Parkinson patients with L-DOPA-induced dyskinesia. Neurochem. 2017;142:756-66.

7. Liepelt I, Reimold M, Maetzler W, Godau J, Reischl G, Gaenslen A, Herbst H, Berg D. Cortical hypometabolism assessed by a metabolic ratio in Parkinson's disease primarily reflects cognitive deterioration-[18F]FDG-PET. Mov Disord. 2010;24:1504-11.

8. Le WD, Xu P, Jankovic J, Jiang H, Appel SH, Smith RG, Vassilatis DK. Mutations in NR4A2 associated with familial Parkinson disease. Nat Genet. 2003;33:85-9.

9. Le W, Pan T, Huang M, Xu P, Xie W, Zhu W, Zhang X, Deng H, Jankovic J. Decreased NURR1 gene expression in patients with Parkinson's disease. J Neurol Sci. 2008:273:29-33.

10. Wang $Y$, Yang Z, Le W. Tiny but mighty: promising roles of MicroRNAs in the diagnosis and treatment of Parkinson's disease. Neurosci Bull. 2017;33:543-51.

11. Heywood WE, Galimberti D, Bliss E, Sirka E, Paterson RW, Magdalinou NK, Carecchio M, Reid E, Heslegrave A, Fenoglio C, et al. Identification of novel CSF biomarkers for neurodegeneration and their validation by a high-throughput multiplexed targeted proteomic assay. Mol Neurodegener. 2015;10:64.

12. Majbour NK, Vaikath NN, van Dijk KD, Ardah MT, Varghese $S$, Vesterager LB, Montezinho LP, Poole S, Safieh-Garabedian B, Tokuda T, et al. Oligomeric and phosphorylated alpha-synuclein as potential CSF biomarkers for Parkinson's disease. Mol Neurodegener. 2016;11:7.

13. Mhyre TR, Boyd JT, Hamill RW, Maguire-Zeiss KA. Parkinson's disease. Subcel Biochem. 2012;65:389-455

14. Lei S, Powers R. NMR metabolomics analysis of Parkinson's disease. Curr Metabolomics. 2013;1:191-209.

15. Sommer A, Winner B, Prots I. The Trojan horse - neuroinflammatory impact of T cells in neurodegenerative diseases. Mol Neurodegener. 2017;12:78.

16. Johansen KK, Wang L, Aasly JO, White LR, Matson WR, Henchcliffe C, Beal MF, Bogdanov M. Metabolomic profiling in LRRK2-related Parkinson's disease. PLoS One. 2009;4:e7551.

17. Bogdanov M, Matson WR, Wang L, Matson T, Saunders-Pullman R, Bressman SS, Flint BM. Metabolomic profiling to develop blood biomarkers for Parkinson's disease. Brain. 2008;131:389-96.

18. Nicholson JK, Connelly J, Lindon JC, Holmes E. Metabonomics: a platform for studying drug toxicity and gene function. Nat Rev Drug Discov. 2002;1:153-61.

19. Chang $\mathrm{KH}$, Cheng ML, Tang HY, Huang CY, Wu YR, Chen CM. Alternations of metabolic profile and kynurenine metabolism in the plasma of Parkinson's disease. Mol Neurobiol. 2018:55:6319-28.

20. Phelan MM, Caamano-Gutierrez E, Gant MS, Grosman RX, Madine J. Using an NMR metabolomics approach to investigate the pathogenicity of amyloid-beta and alpha-synuclein. Metabolomics. 2017;13:151.

21. Shukla AK, Ratnasekhar C, Pragya P, Chaouhan HS, Patel DK, Chowdhuri DK, Mudiam MKR. Metabolomic analysis provides insights on Paraquat- induced Parkinson-like symptoms in Drosophila melanogaster. Mol Neurobiol. 2016:53:254-69.

22. Luan H, Wang X, Cai Z. Mass spectrometry-based metabolomics: targeting the crosstalk between gut microbiota and brain in neurodegenerative disorders. Mass Spectrom Rev. 2017.

23. Leenders J, Frederich $M$, de Tullio P. Nuclear magnetic resonance: a key metabolomics platform in the drug discovery process. Drug Discov Today Technol. 2015;13:39-46.

24. Grimes JH, O'Connell TM. The application of micro-coil NMR probe technology to metabolomics of urine and serum. J Biomol NMR. 2011;49:297-305.

25. Griffin JL. Metabonomics: NMR spectroscopy and pattern recognition analysis of body fluids and tissues for characterisation of xenobiotic toxicity and disease diagnosis. Curr Opin Chem Biol. 2003;7:648-54.

26. Bingol K, Bruschweiler R. Deconvolution of chemical mixtures with high complexity by NMR consensus trace clustering. Anal Chem. 2011:83:7412-7.

27. Walker LR, Hoyt DW, Walker SM 2nd, Ward JK, Nicora CD, Bingol K. Unambiguous metabolite identification in high-throughput metabolomics by hybrid 1D (1) H NMR/ESI MS(1) approach. Magn Reson Chem. 2016;54:998-1003.

28. Smart KF, Aggio RB, Van Houtte JR, Villas-Boas SG. Analytical platform for metabolome analysis of microbial cells using methyl chloroformate derivatization followed by gas chromatography-mass spectrometry. Nat Protoc. 2010;5:1709-29.

29. Peterson AC, Balloon AJ, Westphall MS, Coon JJ. Development of a GC/ quadrupole-Orbitrap mass spectrometer, part II: new approaches for discovery metabolomics. Anal Chem. 2014:86:10044-51.

30. Izadmanesh Y, Garreta-Lara E, Ghasemi JB, Lacorte S, Matamoros V, Tauler R. Chemometric analysis of comprehensive two dimensional gas chromatography-mass spectrometry metabolomics data. J Chromatogr A. 2017; 1488:113-25

31. Dunn WB, Broadhurst D, Begley P, Zelena E, Francis-Mclntyre S, Anderson N, Brown M, Knowles JD, Halsall A, Haselden JN, et al. Procedures for largescale metabolic profiling of serum and plasma using gas chromatography and liquid chromatography coupled to mass spectrometry. Nat Protoc. 2011;6:1060-83.

32. Lotti C, Rubert J, Fava F, Tuohy K, Mattivi F, Vrhovsek U. Development of a fast and cost-effective gas chromatography-mass spectrometry method for the quantification of short-chain and medium-chain fatty acids in human biofluids. Anal Bioanal Chem. 2017:409:5555-67.

33. He L, Prodhan MAI, Yuan F, Yin X, Lorkiewicz PK, Wei X, Feng W, McClain C, Zhang $X$. Simultaneous quantification of straight-chain and branched-chain short chain fatty acids by gas chromatography mass spectrometry. $J$ Chromatogr B. 2018;1092:359-67.

34. Wu Y, Li Y, Jia Y, Wei C, Xu H, Guo R, Li Y, Jia J, Qi X, Gao X. Imbalance in amino acid and purine metabolisms at the hypothalamus in inflammationassociated depression by GC-MS. Mol BioSyst. 2017:13:2715-28.

35. Krone N, Hughes BA, Lavery GG, Stewart PM, Arlt W, Shackleton CHL. Gas chromatography/mass spectrometry (GC/MS) remains a pre-eminent discovery tool in clinical steroid investigations even in the era of fast liquid chromatography tandem mass spectrometry (LC/MS/MS). J Steroid Biochem Mol Biol. 2010;121:496-504

36. Stoll DR, Harmes DC, Staples GO, Potter OG, Dammann CT, Guillarme D, Beck A. Development of comprehensive online two-dimensional liquid chromatography/mass spectrometry using hydrophilic interaction and reversed-phase separations for rapid and deep profiling of therapeutic antibodies. Anal Chem. 2018;90:5923-9.

37. Buszewski B, Noga S. Hydrophilic interaction liquid chromatography (HILIC)-a powerful separation technique. Anal Bioanal Chem. 2012:402:231-47.

38. Wang S, Zhou L, Wang Z, Shi X, Xu G. Simultaneous metabolomics and lipidomics analysis based on novel heart-cutting two-dimensional liquid chromatography-mass spectrometry. Anal Chim Acta. 2017;966:34-40.

39. Zhou J, Yin Y. Strategies for large-scale targeted metabolomics quantification by liquid chromatography-mass spectrometry. Analyst. 2016;141:6362-73.

40. van der Greef J, van Wietmarschen H, van Ommen B, Verheij E. Looking back into the future: 30 years of metabolomics at TNO. Mass Spectrom Rev. 2013;32:399-415.

41. Cajka T, Fiehn O. Toward merging untargeted and targeted methods in mass spectrometry-based metabolomics and Lipidomics. Anal Chem. 2016; 88:524-45.

42. Zhou J, Liu H, Liu Y, Liu J, Zhao X, Yin Y. Development and evaluation of a parallel reaction monitoring strategy for large-scale targeted metabolomics quantification. Anal Chem. 2016;88:4478-86. 
43. Goldstein DS, Holmes C, Sharabi Y. Cerebrospinal fluid biomarkers of central catecholamine deficiency in Parkinson's disease and other synucleinopathies. Brain. 2012;135:1900-13.

44. Abe T, Isobe C, Murata T, Sato C, Tohgi H. Alteration of 8-hydroxyguanosine concentrations in the cerebrospinal fluid and serum from patients with Parkinson's disease. Neurosci Lett. 2003:336:105-8.

45. Ascherio A, LeWitt PA, Xu K, Eberly S, Watts A, Matson WR, Marras C, Kieburtz K, Rudolph A, Bogdanov MB, et al. Urate as a predictor of the rate of clinical decline in Parkinson disease. Arch Neurol. 2009;66:1460-8.

46. Cheng FC, Kuo JS, Chia LG, Dryhurst G. Elevated 5-S-cysteinyldopamine/ homovanillic acid ratio and reduced homovanillic acid in cerebrospinal fluid: possible markers for and potential insights into the pathoetiology of Parkinson's disease. J Neural Transm (Vienna). 1996;103:433-46.

47. LeWitt $P$, Schultz L, Auinger P, Lu M, Parkinson Study Group DI. CSF xanthine, homovanillic acid, and their ratio as biomarkers of Parkinson's disease. Brain Res. 2011;1408:88-97.

48. Stoessel D, Stellmann JP, Willing A, Behrens B, Rosenkranz SC, Hodecker SC, Sturner KH, Reinhardt S, Fleischer S, Deuschle C, et al. Metabolomic profiles for primary progressive multiple sclerosis stratification and disease course monitoring. Front Hum Neurosci. 2018;12:226.

49. Roede JR, Uppal K, Park Y, Lee K, Tran V, Walker D, Strobel FH, Rhodes SL, Ritz B, Jones DP. Serum metabolomics of slow vs rapid motor progression Parkinson's disease: a pilot study. PLoS One. 2013;8:e77629.

50. Trupp $M$, Jonsson $P$, Ohrfelt A, Zetterberg H, Obudulu O, Malm L, Wuolikainen A, Linder J, Moritz T, Blennow K, et al. Metabolite and peptide levels in plasma and CSF differentiating healthy controls from patients with newly diagnosed Parkinson's disease. J Parkinsons Dis. 2014;4:549-60.

51. Öhman A, Forsgren L. NMR metabonomics of cerebrospinal fluid distinguishes between Parkinson's disease and controls. Neurosci Lett. 2015;594:36-9.

52. Stoessel D, Schulte C, Teixeira Dos Santos MC, Scheller D, Rebollo-Mesa I, Deuschle C, Walther D, Schauer N, Berg D, Nogueira da Costa A, Maetzler W. Promising Metabolite Profiles in the Plasma and CSF of Early Clinical Parkinson's Disease. Front Aging Neurosci. 2018;10:51.

53. Oxenkrug G, van der Hart M, Roeser J, Summergrad P. Peripheral Tryptophan Kynurenine Metabolism Associated with Metabolic Syndrome is Different in Parkinson's and Alzheimer's Diseases. Endocrinol Diabetes Metab J. 2017:1.

54. Han W, Sapkota S, Camicioli R, Dixon RA, Li L. Profiling novel metabolic biomarkers for Parkinson's disease using in-depth metabolomic analysis. Mov Disord. 2017;32:1720-8.

55. Cipriani S, Chen X, Schwarzschild MA. Urate: a novel biomarker of Parkinson's disease risk, diagnosis and prognosis. Biomark Med. 2010;4:701-12.

56. Vieru E, Koksal A, Mutluay B, Dirican AC, Altunkaynak Y, Baybas S. The relation of serum uric acid levels with L-Dopa treatment and progression in patients with Parkinson's disease. Neurol Sci. 2016;37:743-7.

57. Schwarzschild MA, Schwid SR, Marek K, Watts A, Lang AE, Oakes D, Shoulson I, Ascherio A, Parkinson Study Group PI, Hyson C, et al. Serum urate as a predictor of clinical and radiographic progression in Parkinson disease. Arch Neurol. 2008;65:716-23.

58. van der Brug MP, Singleton A, Gasser T, Lewis PA. Parkinson's disease: From human genetics to clinical trials. Sci Transl Med. 2015;7:305ps20.

59. Scheperjans F, Pekkonen E, Kaakkola S, Auvinen P. Linking smoking, coffee, urate, and Parkinson's disease - a role for gut microbiota? J Parkinsons Dis. 2015:5:255-62.

60. Jafari S, Etminan M, Aminzadeh F, Samii A. Head injury and risk of Parkinson disease: a systematic review and meta-analysis. Mov Disord. 2013:28:1222-9.

61. Fiandaca M, Gross T, Johnson T, Hu M, Evetts S, Wade-Martins R, MerchantBorna K, Bazarian J, Cheema A, Mapstone M, Federoff H. Potential metabolomic linkage in blood between Parkinson's disease and traumatic brain injury. Metabolites. 2018;8:50.

62. Sato S, Mizuno Y, Hattori N. Urinary 8-hydroxydeoxyguanosine levels as a biomarker for progression of Parkinson disease. Neurology. 2005;64:1081-3.

63. Bolner A, Pilleri M, De Riva V, Nordera GP. Plasma and urinary HPLC-ED determination of the ratio of 8-OHdG/2-dG in Parkinson's disease. Clin Lab. 2011:57:859-66.

64. Hirayama M, Nakamura T, Watanabe H, Uchida K, Hama T, Hara T, Niimi Y, Ito M, Ohno K, Sobue G. Urinary 8-hydroxydeoxyguanosine correlate with hallucinations rather than motor symptoms in Parkinson's disease. Parkinsonism Relat Disord. 2011;17:46-9.

65. Gmitterova K, Heinemann U, Gawinecka J, Varges D, Ciesielczyk B, Valkovic P, Benetin J, Zerr I. 8-OHdG in cerebrospinal fluid as a marker of oxidative stress in various neurodegenerative diseases. Neurodegener Dis. 2009;6:263-9.
66. Alam ZI, Jenner A, Daniel SE, Lees AJ, Cairns N, Marsden CD, Jenner P, Halliwell B. Oxidative DNA damage in the parkinsonian brain: an apparent selective increase in 8-Hydroxyguanine levels in substantia Nigra. J Neurochem. 2002:69:1196-203.

67. Sanchez-Ramos JR, Overvik E, Ames BN. A marker of oxyradical-mediated DNA damage (8-hydroxy-2'deoxyguanosine) is increased in nigro-striatum of Parkinson's disease brain. Neurodegeneration. 1994;3:197-204.

68. Kikuchi A, Takeda A, Onodera H, Kimpara T, Hisanaga K, Sato N, Nunomura A, Castellani RJ, Perry G, Smith MA, Itoyama Y. Systemic increase of oxidative nucleic acid damage in Parkinson's disease and multiple system atrophy. Neurobiol Dis. 2002;9:244-8.

69. Luan H, Liu L-F, Tang Z, Mok VCT, Li M, Cai Z. Elevated excretion of biopyrrin as a new marker for idiopathic Parkinson's disease. Parkinsonism Relat Disord. 2015;21:1371-2

70. Luan H, Liu LF, Meng N, Tang Z, Chua KK, Chen LL, Song JX, Mok VC, Xie LX, Li M, Cai Z. LC-MS-based urinary metabolite signatures in idiopathic Parkinson's disease. J Proteome Res. 2015;14:467-78.

71. Luan H, Liu LF, Tang Z, Zhang M, Chua KK, Song JX, Mok VC, Li M, Cai Z. Comprehensive urinary metabolomic profiling and identification of potential noninvasive marker for idiopathic Parkinson's disease. Sci Rep. 2015;5:13888.

72. Lee W, Park NH, Ahn TB, Chung BC, Hong J. Profiling of a wide range of neurochemicals in human urine by very-high-performance liquid chromatography-tandem mass spectrometry combined with in situ selective derivatization. J Chromatogr A. 2017;1526:47-57.

73. Mulak A, Bonaz B. Brain-gut-microbiota axis in Parkinson's disease. World J Gastroenterol. 2015;21:10609-20.

74. Lin A, Zheng W, He Y, Tang W, Wei X, He R, Huang W, Su Y, Huang Y, Zhou $\mathrm{H}$, Xie H. Gut microbiota in patients with Parkinson's disease in southern China. Parkinsonism Relat Disord. 2018:53:82-8.

75. Sun MF, Shen YQ. Dysbiosis of gut microbiota and microbial metabolites in Parkinson's disease. Ageing Res Rev. 2018;45:53-61.

76. Zierer J, Jackson MA, Kastenmuller G, Mangino M, Long T, Telenti A Mohney RP, Small KS, Bell JT, Steves CJ, et al. The fecal metabolome as a functional readout of the gut microbiome. Nat Genet. 2018;50:790-5.

77. Yu LM, Zhao KJ, Wang SS, Wang X, Lu B. Gas chromatography/mass spectrometry based metabolomic study in a murine model of irritable bowel syndrome. World J Gastroenterol. 2018;24:894-904.

78. Raman M, Ahmed I, Gillevet PM, Probert CS, Ratcliffe NM, Smith S, Greenwood R, Sikaroodi M, Lam V, Crotty P, et al. Fecal microbiome and volatile organic compound metabolome in obese humans with nonalcoholic fatty liver disease. Clin Gastroenterol Hepatol. 2013;11:868-75 e1-3.

79. De Angelis M, Piccolo M, Vannini L, Siragusa S, De Giacomo A, Serrazzanetti DI, Cristofori F, Guerzoni ME, Gobbetti M, Francavilla R. Fecal microbiota and metabolome of children with autism and pervasive developmental disorder not otherwise specified. PLoS One. 2013:8:e76993.

80. Unger MM, Spiegel J, Dillmann KU, Grundmann D, Philippeit H, Burmann J, Fassbender K, Schwiertz A, Schafer KH. Short chain fatty acids and gut microbiota differ between patients with Parkinson's disease and agematched controls. Parkinsonism Relat Disord. 2016;32:66-72.

81. Soret R, Chevalier J, De Coppet P, Poupeau G, Derkinderen P, Segain JP, Neunlist M. Short-chain fatty acids regulate the enteric neurons and control gastrointestinal motility in rats. Gastroenterology. 2010;138:1772-82.

82. Rango M. Parkinson's disease: in vivo brain metabolomics by MRS. Int Rev Neurobiol. 2015;122:81-94.

83. Clarke CE, Lowry M, Horsman A. Unchanged basal ganglia N-acetylaspartate and glutamate in idiopathic Parkinson's disease measured by proton magnetic resonance spectroscopy. Mov Disord. 2004;12:297-301.

84. Rango M, Bonifati C, Bresolin N. Parkinson's disease and brain mitochondrial dysfunction: a functional phosphorus magnetic resonance spectroscopy study. J Cereb Blood Flow Metab. 2006;26:283-90.

85. Borghammer $\mathrm{P}$, Chakravarty M, Jonsdottir KY, Sato N, Matsuda H, Ito K, Arahata Y, Kato T, Gjedde A. Cortical hypometabolism and hypoperfusion in Parkinson's disease is extensive: probably even at early disease stages. Brain Struct Funct. 2010;214:303-17.

86. Rango M, Bonifati C, Bresolin N. Parkinson's disease and brain mitochondrial dysfunction: a functional phosphorus magnetic resonance spectroscopy study. J Cereb Blood Flow Metab. 2006;26:283-90.

87. Tedeschi G, Litvan I, Bonavita S, Bertolino A, Lundbom N, Patronas NJ, Hallett M. Proton magnetic resonance spectroscopic imaging in progressive supranuclear palsy, Parkinson's disease and corticobasal degeneration. Brain. 1997;120(Pt 9):1541. 
88. Davie CA, Pirtosek Z, Barker GJ, Kingsley DP, Miller PH, Lees AJ. Magnetic resonance spectroscopic study of parkinsonism related to boxing. J Neurol Neurosurg Psychiatry. 1995;58:688-91.

89. Wood PL, Tippireddy S, Feriante J, Woltjer RL. Augmented frontal cortex diacylglycerol levels in Parkinson's disease and Lewy body disease. PLoS One. 2018;13:e0191815.

90. Rappley I, Myers DS, Milne SB, Ivanova PT, Lavoie MJ, Brown HA, Selkoe DJ. Lipidomic profiling in mouse brain reveals differences between ages and genders, with smaller changes associated with alpha-synuclein genotype. J Neurochem. 2009;111:15-25.

91. Chen X, Xie C, Sun L, Ding J, Cai H. Longitudinal metabolomics profiling of Parkinson's disease-related alpha-Synuclein A53T transgenic mice. PLoS One. 2015;10:e0136612.

92. Anandhan A, Lei S, Levytskyy R, Pappa A, Panayiotidis MI, Cerny RL, Khalimonchuk O, Powers R, Franco R. Glucose metabolism and AMPK signaling regulate dopaminergic cell death induced by gene (alpha-Synuclein)environment (Paraquat) interactions. Mol Neurobiol. 2017;54:3825-42.

93. Poliquin PO, Chen J, Cloutier M, Trudeau LE, Jolicoeur M. Metabolomics and in-silico analysis reveal critical energy deregulations in animal models of Parkinson's disease. PLoS One. 2013;8:e69146.

94. Tanner CM, Kamel F, Ross GW, Hoppin JA, Goldman SM, Korell M, Marras C, Bhudhikanok GS, Kasten M, Chade AR. Rotenone, Paraquat, and Parkinson's disease. Environ Health Perspect. 2011;119:866-72.

95. Martinez TN, Greenamyre JT. Toxin models of mitochondrial dysfunction in Parkinson's disease. Antioxid Redox Signal. 2012;16:920-34.

96. Simola N, Morelli M, Carta AR. The 6-Hydroxydopamine model of parkinson's disease. Neurotox Res. 2007;11:151-67.

97. Graham SF, Rey NL, Yilmaz A, Kumar P, Madaj Z, Maddens M, Bahado-Singh RO, Becker K, Schulz E, Meyerdirk LK, et al. Biochemical profiling of the brain and blood metabolome in a mouse model of prodromal Parkinson's disease reveals distinct metabolic profiles. J Proteome Res. 2018;17:2460-9.

98. Lei S, Zavala-Flores L, Garcia-Garcia A, Nandakumar R, Huang Y, Madayiputhiya N, Stanton RC, Dodds ED, Powers R, Franco R. Alterations in energy/redox metabolism induced by mitochondrial and environmental toxins: a specific role for glucose-6-phosphatedehydrogenase and the pentose phosphate pathway in paraquat toxicity. ACS Chem Biol. 2014;9:2032-48.

99. Tyurina YY, Polimova AM, Maciel E, Tyurin VA, Kapralova VI, Winnica DE, Vikulina AS, Domingues MR, McCoy J, Sanders LH, et al. LC/MS analysis of cardiolipins in substantia nigra and plasma of rotenone-treated rats: implication for mitochondrial dysfunction in Parkinson's disease. Free Radic Res. 2015;49:681-91.

100. Farmer K, Smith CA, Hayley S, Smith J. Major alterations of phosphatidylcholine and Lysophosphotidylcholine lipids in the substantia Nigra using an early stage model of Parkinson's disease. Int J Mol Sci. 2015;16:18865-77.

101. Schulte EC, Altmaier E, Berger HS, Do KT, Kastenmuller G, Wahl S, Adamski J, Peters A, Krumsiek J, Suhre K, et al. Alterations in lipid and inositol metabolisms in two dopaminergic disorders. PLoS One. 2016;11:e0147129.

102. Saiki S, Hatano T, Fujimaki M, Ishikawa Kl, Mori A, Oji Y, Okuzumi A, Fukuhara T, Koinuma T, Imamichi Y, et al. Decreased long-chain acylcarnitines from insufficient beta-oxidation as potential early diagnostic markers for Parkinson's disease. Sci Rep. 2017;7:7328.

103. Andersen AD, Blaabjerg M, Binzer $M$, Kamal $A$, Thagesen $H$, Kjaer TW, Stenager E, Gramsbergen JBP. Cerebrospinal fluid levels of catecholamines and its metabolites in Parkinson's disease: effect of I-DOPA treatment and changes in levodopa-induced dyskinesia. J Neurochem. 2017;141:614-25.

104. Golovko MY, Barcelo-Coblijn G, Castagnet PI, Austin S, Combs CK, Murphy EJ. The role of alpha-synuclein in brain lipid metabolism: a downstream impact on brain inflammatory response. Mol Cell Biochem. 2009;326:55-66.

105. Powers R, Lei S, Anandhan A, Marshall DD, Worley B, Cerny RL, Dodds ED, Huang Y, Panayiotidis MI, Pappa A, Franco R. Metabolic investigations of the molecular mechanisms associated with Parkinson's disease. Meta. 2017;7.

106. Jia W, Xie G, Jia W. Bile acid-microbiota crosstalk in gastrointestinal inflammation and carcinogenesis. Nat Rev Gastroenterol Hepatol. 2018; 15:111-28.

107. Castrocaldas M, Carvalho AN, Rodrigues E, Henderson CJ, Wolf CR, Rodrigues CMP, Gama MJ. Tauroursodeoxycholic acid prevents MPTPinduced dopaminergic cell death in a mouse model of Parkinson's disease. Mol Neurobiol. 2012:46:475-86.

108. Chiasserini D, Paciotti S, Eusebi P, Persichetti E, Tasegian A, Kurzawa-Akanbi M, Chinnery PF, Morris CM, Calabresi P, Parnetti L, Beccari T. Selective loss of glucocerebrosidase activity in sporadic Parkinson's disease and dementia with Lewy bodies. Mol Neurodegener. 2015;10:15.

109. Quansah E, Peelaerts W, Langston JW, Simon DK, Colca J, Brundin P. Targeting energy metabolism via the mitochondrial pyruvate carrier as a novel approach to attenuate neurodegeneration. Mol Neurodegener. 2018;13:28.

110. Mayer EA, Tillisch K, Gupta A. Gut/brain axis and the microbiota. J Clin Invest. 2015;125:926-38.

111. Burte F, Houghton D, Lowes H, Pyle A, Nesbitt S, Yarnall A, Yu-Wai-Man P, Burn DJ, Santibanez-Koref M, Hudson G. Metabolic profiling of Parkinson's disease and mild cognitive impairment. Mov Disord. 2017;32:927-32.

112. Hatano T, Saiki S, Okuzumi A, Mohney RP, Hattori N. Identification of nove biomarkers for Parkinson's disease by metabolomic technologies. J Neurol Neurosurg Psychiatry. 2016;87:295-301.

113. Ahmed SS, Santosh W, Kumar S, Christlet HT. Metabolic profiling of Parkinson's disease: evidence of biomarker from gene expression analysis and rapid neural network detection. J Biomed Sci. 2009;16:63.

114. Lu Z, Wang J, Li M, Liu Q, Wei D, Yang M, Kong L. (1)H NMR-based metabolomics study on a goldfish model of Parkinson's disease induced by 1-methyl-4-phenyl-1,2,3,6-tetrahydropyridine (MPTP). Chem Biol Interact. 2014;223:18-26

115. Zheng $H$, Zhao L, Xia H, Xu C, Wang D, Liu K, Lin L, Li X, Yan Z, Gao H. NMR-based metabolomics reveal a recovery from metabolic changes in the striatum of 6-OHDA-induced rats treated with basic fibroblast growth factor Mol Neurobiol. 2016;53:6690-7.

116. Li XZ, Zhang SN, Lu F, Liu CF, Wang Y, Bai Y, Wang N, Liu SM. Cerebral metabonomics study on Parkinson's disease mice treated with extract of Acanthopanax senticosus harms. Phytomedicine. 2013;20:1219-29.
Ready to submit your research? Choose BMC and benefit from:

- fast, convenient online submission

- thorough peer review by experienced researchers in your field

- rapid publication on acceptance

- support for research data, including large and complex data types

- gold Open Access which fosters wider collaboration and increased citations

- maximum visibility for your research: over $100 \mathrm{M}$ website views per year

At $\mathrm{BMC}$, research is always in progress.

Learn more biomedcentral.com/submissions 